Chapter 10

\title{
Imaging Findings of Gastric Carcinoma
}

\author{
Eriko Maeda, Masaaki Akahane, Kuni Ohtomo, \\ Keisuke Matsuzaka and Masashi Fukayama \\ Additional information is available at the end of the chapter \\ http://dx.doi.org/10.5772/52076
}

\section{Introduction}

Surgery is the only certain treatment for gastric carcinoma, so early detection and accurate staging is a key to successive treatment and mortality reduction. In this chapter, we would like to explain radiologic imaging of gastric carcinoma by

1. Reviewing the evidences for gastric carcinoma screening

2. Demonstrating the TNM classification of gastric carcinoma and the relevant imaging findings for each stages and

3. Introducing unusual imaging findings of gastric carcinoma and differential diagnoses.

\subsection{Gastric carcinoma screening}

Gastric carcinoma is the fourth most common cancer worldwide, behind lung, breast and colorectal carcinomas, and is the second leading cause of death in both sexes worldwide and in Asia [1, 2]. There is about twice male predominance.Gastric carcinoma is particularly common in countries such as Korea (incidence 62.2 per 100,000 males; mortality 22.8 per 100,000 males), Japan (46.8; 20.5), China (41.3; 30.5), Chile (27.3; 23.1), Russia (26.9; 24.0) but not as common in a large part of western societies such as the United States $(5.7 ; 2.7)$ and United Kingdom $(8.0 ; 4.8)$ [1].

The high mortality is mainly due to late presentation, therefore early detection and treatment is an important way to reduce death from gastric cancer [2].There are four major methods for screening gastric carcinoma; fluoroscopy, endoscopy, serum pepsinogen testing, and Helicobacter pylori antibody testing [3]. Because of a large difference in burden of gastric carcinoma among nations, benefit of gastric cancer screening cannot be debated on the same 
ground for societies throughout the world. However, there have been no randomized trials evaluating the impact of screening on mortality from gastric carcinoma [2,3].For societies where gastric carcinoma is uncommon, National Cancer Institute of the United States state that for screening would not result in a decrease in mortality fromgastric carcinoma [4].

In Japan, there is a government-sponsored mass screening program with barium meal fluorography. Participants are recommended to undergo endoscopy of the upper gastrointestinal tract when positive findings are detected at fluorography. Asymptomatic individuals older than 40 years are eligible for this program, but only around $20 \%$ of the eligible subjects actually participates the program [3]. Most case-control studies from Japan show a 40-60\% decrease in mortality from gastric carcinoma in the subjects who participated the program [2,5-8]. In contrast, Japanese prospective series setting death from gastric cancer as an endpoint have inconsistent results [2,9-13]. Even in combination with serum pepsinogen, a large Japanese study screening 17,647 men aged 40-60 years the positive predictive value over the 7 -year period was $0.85 \%$ [14].Thus even in societies with high incidence, identification of high-risk groups that benefit from screening may be necessary to perform cost-effective screening.The subgroups might include elderly patients with atrophic gastritis or pernicious anemia, patients with partial gastrectomy, patients with Epstein-Barr virus associated gastric carcinoma or history of multiple carcinomas, patients with the diagnosis of sporadic adenomas, familial adenomatous polyposis or hereditary nonpolyposis colon cancer [15-19].

Endoscopy has advantage over fluoroscopy, especially in detection of flat and non-ulcerative lesions. A Japanese study comparing finding ratio of gastric carcinoma with fluoroscopy and endoscopy reports 2.7 to 4.6-times higher ratio for endoscopy [20]. However, effective screening with endoscopy relies on the skill of the endoscopists and availability of endoscopes, and it is likely to be unfeasible to perform mass screening using endoscopy.

\section{TNM classification of gastric carcinomas and the relevant imaging findings}

Owing to recent advances in CT technology, we have been able to visualize early carcinomas and to stage tumors with considerable accuracy, with the use of appropriate contrast technique and effervescent agent or water [21]. Recent CT with conventional transverse images, multiplanar reconstruction (MPR) images and virtual endoscopy can detect gastric carcinomas efficiently with the detection rates of $91 \%, 96 \%$ and $98 \%$, respectively [22].

Gastric carcinomas appear as a focal area of mural thickening with or without ulceration, as a polypoid lesion, or as generalized mural thickening. Lesions occurring in the antrum, in the body, and in the fundus comprise $30 \%$ of all gastric carcinomas respectively, and the remaining $10 \%$ involve the whole stomach [23].

CT criteria for T staging of gastric carcinoma is as follows [22].

$\mathrm{T} 1$ lesion $=$ focal thickening of the inner layer, almost well enhanced, and has visible lowattenuation-strip outer layer of gastric wall and clear fat plane around tumor 
T2 lesion = focal or diffuse thickening of the wall with transmural enhancement, almost well enhanced, and has smooth outer wall border and clear fat plane around tumor

T3 lesion $=$ transmural tumor with irregular or nodular outer border and/or perigastric fat infiltration

T4 lesion = Obliteration of fat plane betweengastric tumor and adjacent organ or invasion of adjacent organ

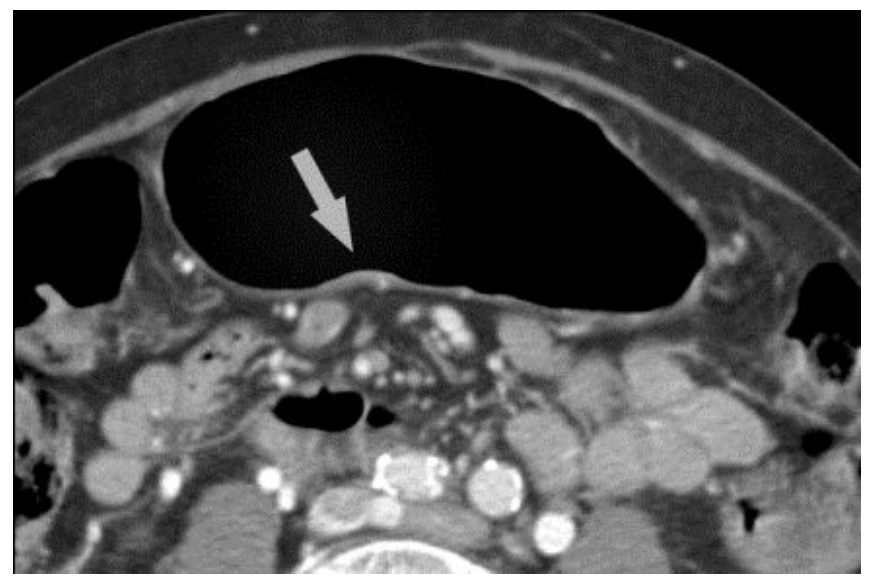

Figure 1. T1a gastric carcinoma in a 76-year old man. Contrast CT shows a subtle thickening and enhancement of the inner layer (arrow) with low-attenuation-strip outer layer of gastric wall and clear fat plane around tumor.

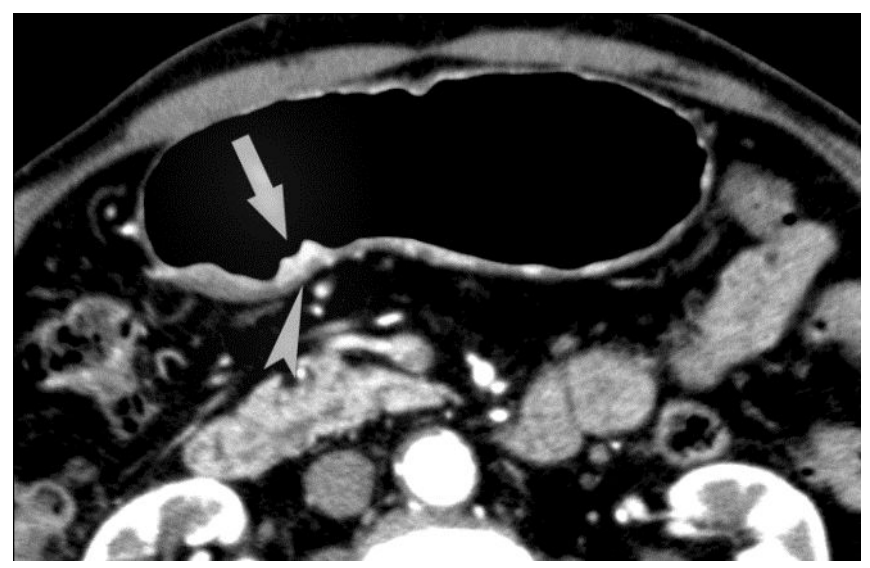

Figure 2. T2 gastric carcinoma in a 82-year old man. Contrast CT shows a well-enhanced focal mural thickening (arrow)andfocal enhancement of the outer layer (arrowhead). The tumor has smooth outer wall border and clear fat plane around tumor. 
Accuracy of CT in T-staging with transverse images only is $73 \%$, but it rises to $89 \%$ with the use of MPR [22]. Therefore it is important to perform appropriate reconstruction techniques in CT diagnosis of gastric carcinoma.

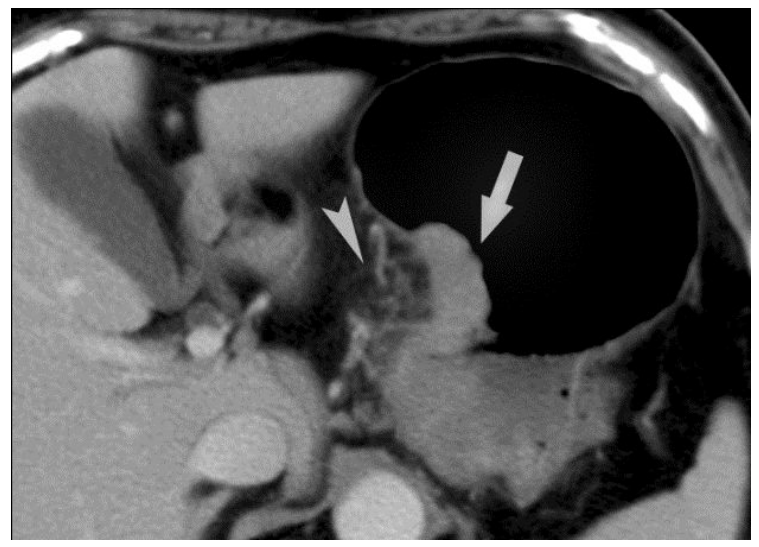

Figure 3. T3 gastric carcinoma in a 64-year old woman. Contrast CT shows a mass in the lesser curvature (arrow), obliterating the outer layer of the stomach. The outer border of the tumor is irregular, and perigastric fat stranding is visualized (arrowhead).

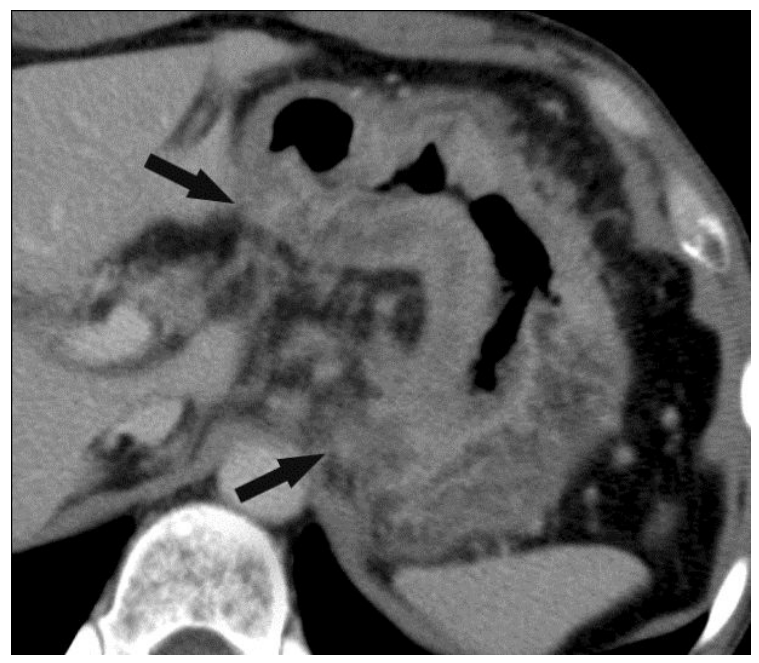

Figure 4. Type IV gastric carcinoma in a 47-yer old man. Contrast CT shows diffuse mural thickening obliterating thefolds and the inner structure of the gastric wall (arrows). The enhancement "running" through the gastric wall is characteristic of scirrhous tumors. 
$\mathrm{T} 1$ tumors are classified into T1a and T1b tumors; a T1a lesion stay within the mucosal layer, while a T1b lesion stay within the submucosal layer (Figure 1).

A T2 tumor infiltrates into the muscularis propria layer and stays within the layer (Figure 2).

A T3 tumor extends over the muscularis propria layer, but its border stays within the subserosal layer (Figure 3).

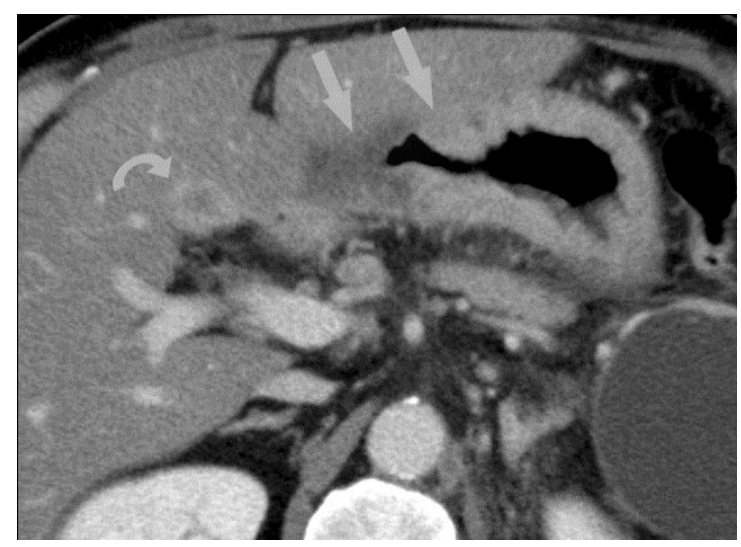

Figure 5. Advanced gastric carcinoma in a 60-year old man. Contrast CT shows diffuse mural thickening of the antrum (arrows). The fat plane between the tumor and the liver is obliterated. Liver metastasis can be found as well (curved arrow).

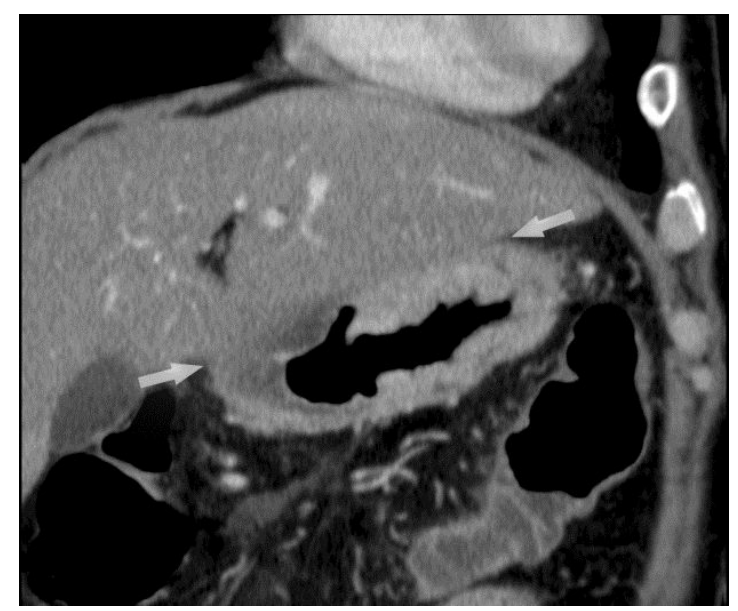

Figure 6. Advanced gastric carcinoma in a 60-year old man (the same patient as Figure 5). The clear and smooth border between the tumor and the liver (between two arrows) can be shown with coronal MPR. This tumor can be staged as T4a. 
T4 tumors are classified into T4a and T4b tumors; A T4a lesion invades the serosa, exposing its surface to the peritoneal cavity in many cases. The tumor is classified as T4b when it invades the adjacent organs, such as the transverse colon, pancreas, spleen, liver and the diaphragm.Signet-ring cell carcinoma, often found at T4a stage, usually manifests as a scirrhous tumor, and appears as diffuse thickening of the gastric wall with obliteration of gastric folds, usually extending from the antrum into the body and fundus (Figure 4) [23].

$\mathrm{T} 4 \mathrm{~b}$ tumor requires resection of adjacent organs with the primary tumor, and discrimination of T4a tumors from "T4b-looking tumor" is an important function of preoperative imaging. An advanced tumor can be recognized as T4a when the fat plane between the tumor and the adjacent organ is visualized, or when the fat plane is invisible or compressed by the tumor, the tumor is considered to be T4a if it has a clear and smooth border (Figures 5-7).MPR in appropriate plane is especially effective in differentiating between T4a and T4b; MPR is reported to improve the specificity without compensation in sensitivity in diagnosis of invasion into the transverse colon or mesocolon and the pancreas [24].

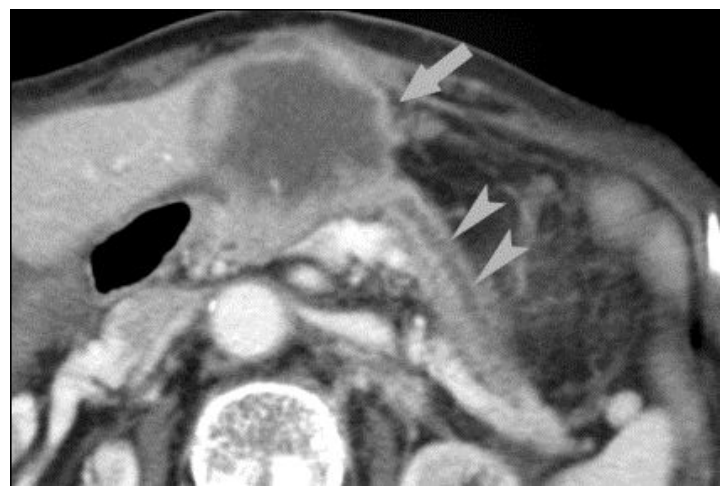

Figure 7. Advanced gastric carcinoma in a 88-year old woman. The tumor extends downwards toward the pancreas (arrow), and causes dilatation of the main pancreatic duct as a result of pancreatic infiltration (arrowheads). This tumor is staged as T4b.

Criteria for $\mathrm{N}$ staging for gastric carcinoma is as follows:

N0 = no lymph nodes involved

$\mathrm{N} 1=$ metastases in 1-2 regional lymph nodes

$\mathrm{N} 2=$ metastases in 3-6 regional lymph nodes

$\mathrm{N} 3 \mathrm{a}=$ metastases in 7-15 regional lymph nodes

$\mathrm{N} 3 \mathrm{~b}=$ metastases in more than 15 regional lymph nodes

Gastric carcinoma is often accompanied with nodal metastases even at relatively earlier stages. Micrometastases and normal-sized metastatic nodes are common in gastric carcinoma, and this makes accurate $\mathrm{N}$ staging difficult. Ring enhancement,inhomogeneous enhancementand 
strong enhancement at arterial phase are known as possible signs of metastases in a normal sized lymph node. Therefore it is important to point out nodes with these atypical findings, even when the node is smaller than $10 \mathrm{~mm}$.Since accurate counting of lymph node metastases is the key to accurate $\mathrm{N}$ staging, active reconstruction with MPR is warranted for accurate measurement and interpretation of conglomerated lymph nodes (Figures 8,9).

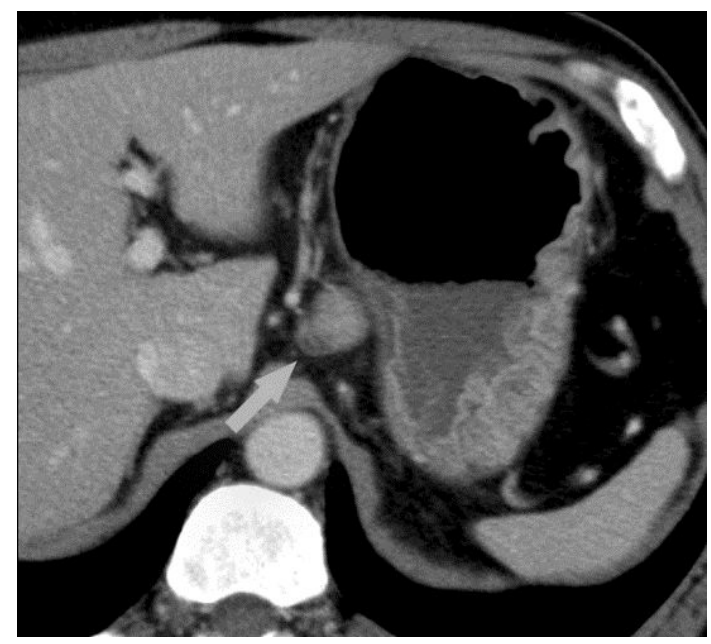

Figure 8. A 50-year old man with gastric carcinoma. Contrast CT shows a mass at the lesser curvature (arrow).

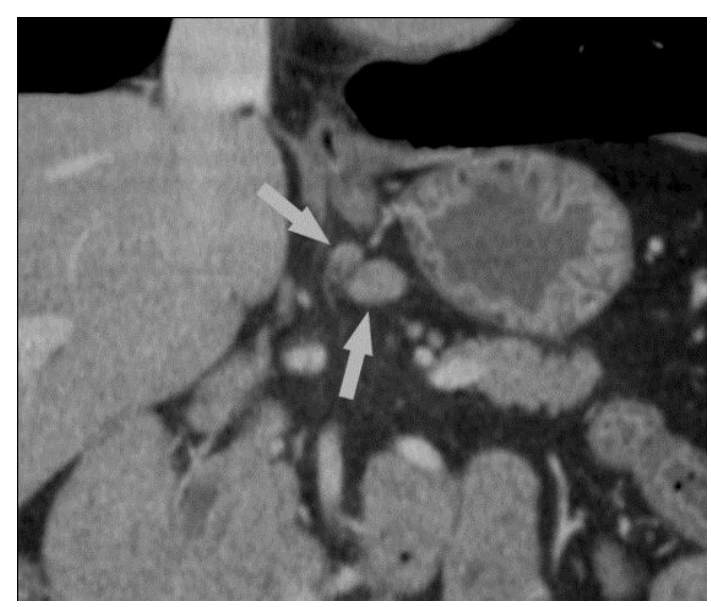

Figure 9. A 50-year old man with gastric carcinoma (the same patient as Figure 8). MPR in the coronal plane shows this mass consists of two lymph nodes. 
The liver is the most common metastatic sites for gastric carcinoma because the gastric veins drain into the hepatic portal system.Metastatic hepatic tumors are often accompanied with ring enhancement at earlier phase, and portal phase in addition to the equilibrium phase. Some tumors lose contrast to the liver parenchyma after the delivery of the contrast material, and we obtain the plane CT images as well in the metastasis survey protocolof our institution.Other common sites for distant metastases include the lungs, adrenal glands, and the ovaries (Krukenberg tumors).Positron emission tomography (PET) with 2-[fluorine-18]fluoro-2-deoxy-d-glucose (FDG) is not appropriated for local tumor staging, but is effective for detection of distant metastases [25].

CT does not have enough sensitivity for detection of peritoneal dissemination. Even with recent 16- or 64-row detector scanners, the sensitivity and specificity of CT diagnosis of peritoneal dissemination are $28.3 \%$ and $98.9 \%$ respectively when definite criteria are adopted, and $50.9 \%$ and $96.2 \%$ when the criteria included the suspicious findings [26]. This report mentions greater tumor size and advanced $\mathrm{T}$ stage as predictive factors for dissemination, and recommends staging laparoscopy for tumors with these factors, even when CT results are negative for peritoneal dissemination. The value of FDG-PET in detection of peritoneal dissemination is still controversial [25].

\section{Unusual imaging findings of gastric carcinoma and differential diagnoses}

Rarely, gastric carcinomas present with gross or psammomatous calcifications. Calcified gastric carcinomas are usually found in mucinous adenocarcinoma; a carcinoma characterized by prominent glandular formations and abundant mucin deposition. Calcifications in mucinous carcinoma are military and punctate $[27,28]$. Rarely, calcification within gastric carcinoma lesion occurs as a result of secretion of parathyroid hormone-like substance [29]. Other reported atypical features of gastric carcinomas include transpyloric spread, giant gastric folds and hypervascular masses [27,30,31].

Epstein-Barr virus associated gastric carcinoma (EBVaGC) is a clinicopathologically and molecularly distinct type of gastric carcinoma.EBV-associated gastric carcinoma (EBVaGC) occurs worldwide, with the reported incidence varying from $1.3 \%$ to $20.1 \%$, affects $70,000-80,000$ people per year (estimate), constituting the largest group of EBV-associated malignancies [16,32,33].EBVaGC is associated with male predominance, location in the proximal stomach, multiplicity and carcinomas affecting remnant stomachs [33,34].Although there are some conflicting evidences, lower rate of lymph node involvement and relatively favorable prognosis is suggested [32,33,35].EBVaGC is associated with two types of histology: lymphoepithelioma (LE) -like type which is almost identical to the subgroup reported as "gastric carcinoma with lymphoid stroma (GCLS)", and ordinary type [36,37].Imaging findings of LE-like type or GCLS is characterized by a large thickness-to-length ratio, and is sometimes accompanied with a bulky portion projecting from the gastric wall [38] (Figure $10)$. 


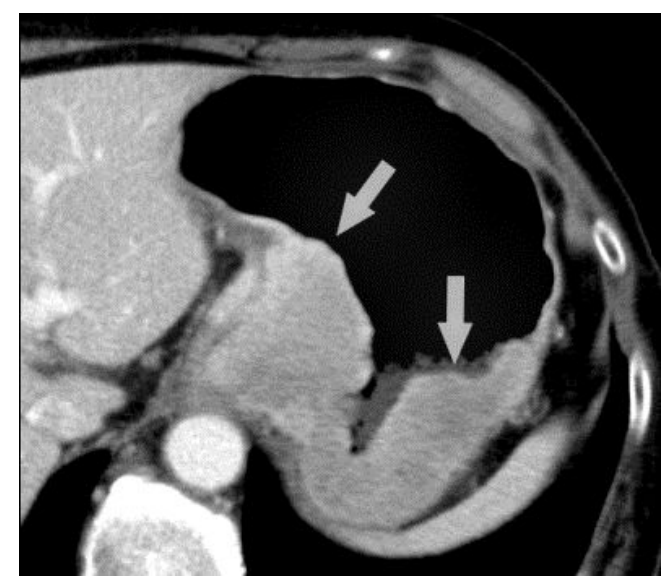

Figure 10. LE-type EBVaGC in a 69-year old woman. Contrast CT shows massive mural thickening involving the gastric fundus and the esophagogastric junction (arrows).

Gastric carcinoma need to be differentiated from other malignant tumors involvingthe stomach, whichincludes carcinoid, carcinosarcoma, lymphoma, mucosa-associated lymphoid tissue lymphoma (MALToma) and gastrointestinal stromal tumor (GIST) (Figures 11-13) [27]. Benign tumors of the stomach include hyperplastic or adenomatous polyps, leiomyoma, schwannoma, lipoma, hemangioma and glomus tumor. Heterotopic pancreas can also be mistaken as a gastric carcinoma (Figure 14).

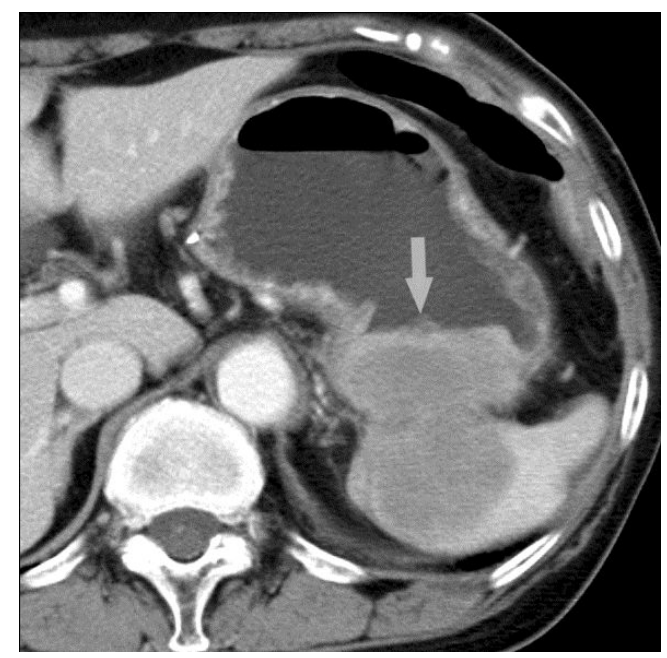

Figure 11. Diffuse large B-cell lymphoma in a 73-year old woman. Contrast CT shows a dumbbell-shaped mass extending from the fundus into the spleen (arrow). 


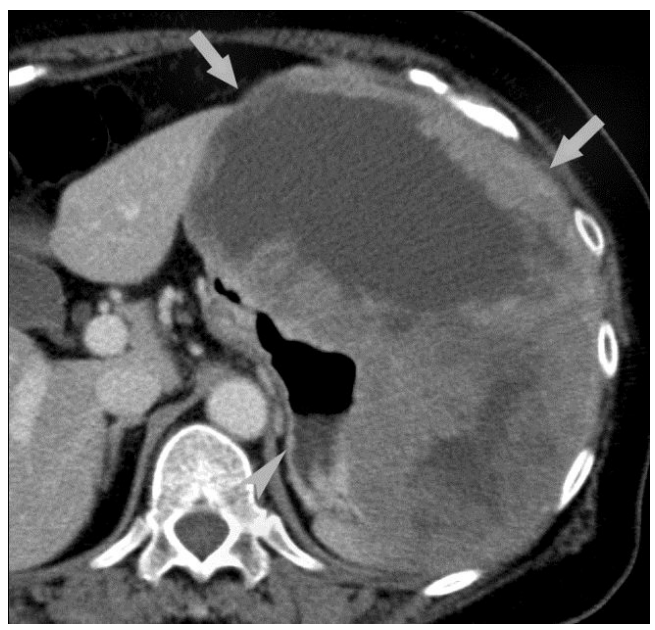

Figure 12. High-grade GIST in a 53-year old man. Contrast CT shows an enormous tumor extending along the outer gastric wall (arrows). The tumor has a smooth border but the enhancement is very heterogeneous, with a large area of necrosis showing homogeneous low attenuation. Note the compressed cavity of the stomach (arrowhead).

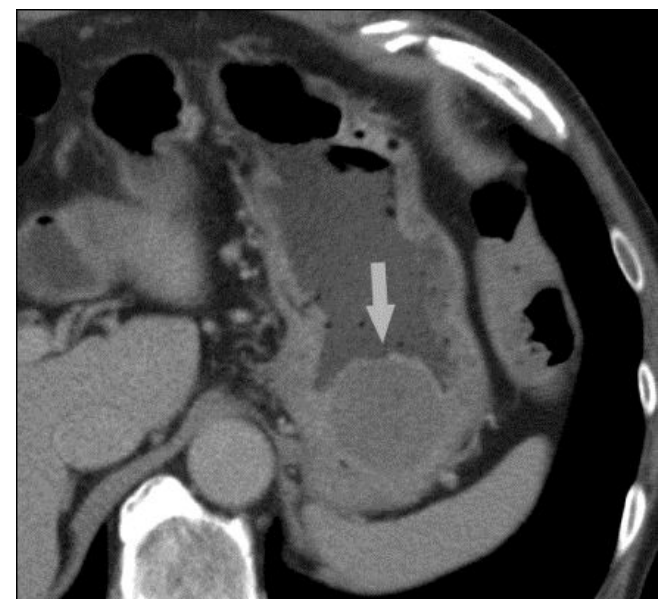

Figure 13. Low-grade GIST in a 74-year old man. Contrast CT shows a smooth round tumor with homogeneous enhancement within the fundus (arrow). 


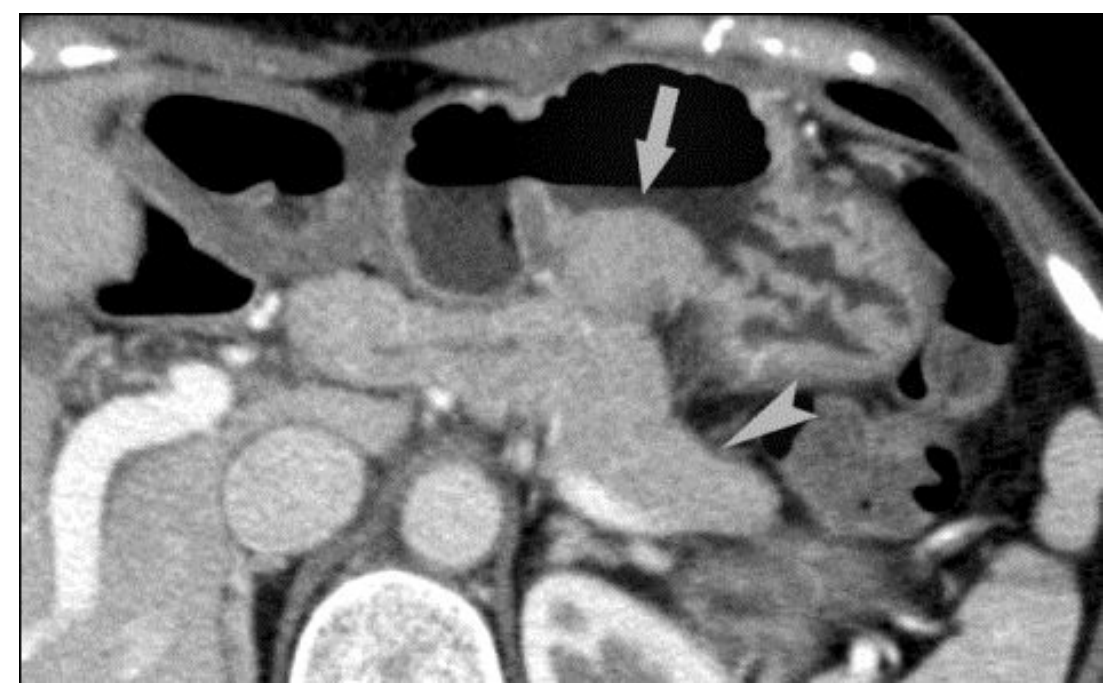

Figure 14. A 46-year old woman with a submucosal mass. Contrast CT shows a mass (arrow) presenting similar enhancement as the pancreas (arrowhead). Heterotopic pancreas was suspected on CT and at endoscopic ultrasonography, and was confirmed by fine-needle biopsy.

\section{Conclusion}

Although early detection is the key to the mortality reduction of gastric carcinoma, the benefit of screening is still under debate even in the societies with high incidence. Recent CT with appropriate reconstruction technique can detect and locally stage gastric carcinomas sufficiently. It remains a challenge to accurately diagnose lymph node metastasis and peritoneal dissemination with imaging. Imaging can also depict unusual manifestationsof gastric carcinomas such as calcification and a large thickness-to-width ratio or projecting mass in EBVaGC.

\section{Author details}

Eriko Maeda ${ }^{1 *}$, Masaaki Akahane ${ }^{1}, K_{\text {Kuni Ohtomo }}{ }^{1}$, Keisuke Matsuzaka ${ }^{2}$ and Masashi Fukayama

*Address all correspondence to: emaeda-tky@umin.ac.jp

1 Department of Radiology, Graduate School of Medicine, University of Tokyo, Japan

2 Department of Pathology, Graduate School of Medicine, University of Tokyo, Japan 


\section{References}

[1] Ferlay, J., Shin, H.R., Bray, F., Forman, D., Mathers, C., \& Parkin, D.M. (2008). GLOBOCAN v1.2, Cancer Incidence and Mortality Worldwide: IARC Cancer Base No. 10Lyon, France: International Agency for Research on Cancer.

[2] Leung, W. K., Wu, M. S., Kakugawa, Y., et al. (2008). Asia Pacific Working Group on Gastric Cancer. Screening for gastric cancer in Asia: current evidenceand practice. Lancet Oncol., 9(3), 279-87.

[3] Hamashima, C., Shibuya, D., Yamazaki, H., et al. (2008). The Japanese guidelines for gastric cancer screening. Jpn J Clin Oncol., 38(4), 259-67.

[4] National Cancer Institute. (2012). Stomach (Gastric) cancer screening (PDQ) cancer. Health Professional, http://cancer.gov/cancertopics/pdq/screening/gastric/ .

[5] Kunisaki, C., Ishino, J., Nakajima, S., et al. (2006). Outcomes of mass screening for gastric carcinoma. Ann Surg Oncol, 13(2), 221-28.

[6] Oshima, A., Hirata, N., Ubukata, T., et al. (1986). Evaluation of a mass screening program for stomach cancer with a case-control study design. Int J Cancer, 38(6), 829-33.

[7] Fukao, A., Tsubono, Y., Tsuji, I., et al. (1995). The evaluation of screening for gastric cancer in Miyagi Prefecture, Japan: a population-based case-control study. Int L Cancer, 60(1), 45-48.

[8] Abe, Y., Mitsushima, T., Nagatani, K., et al. (1995). Epidemiological evaluation of the protective effect for dying of stomach cancer by screening programme for stomach cancer with applying a method of case-control study-a study of an efficient screening programme for stomach cancer. Nippon Shokakibyo Gakkai Zasshi, 92(5), 836-45.

[9] Oshima, A., Hanai, A., \& Fujimoto, I. (1979). Evaluation of a mass screening program for stomach cancer. Natl Cancer Inst Monogr., 53, 181-86.

[10] Hisamichi, S., \& Sugawara, N. (1984). Mass screening for gastric cancer by X-ray examination. Jpn J Clin Oncol., 14(2), 211-13.

[11] Inaba, S., Hirayama, H., Nagata, C., et al. (1999). Evaluation of a screening program on reduction of gastric cancer mortality in Japan: preliminary results from a cohort study. Prev Med., 20(2), 9-102.

[12] Mizoue, T., Yoshimura, T., Tokui, N., et al. (2003). Prospective study of screening for stomach cancer in Japan. Int J Cancer:, 106(1), 103-07.

[13] Lee, K. J., Inoue, M., Otani, T., et al. (2006). Gastric cancer screening and subsequent risk of gastric cancer: a large-scale population-based cohort study, with a 13-year follow up in Japan. Int J Cancer, 118(9), 2315-21. 
[14] Ohata, H., Oka, M., Yanaoka, K., et al. (2005). Gastric cancer screening of a high-risk population in Japan using serum pepsinogen an barium digital radiography. Cancer Sci., 96(10), 713-20.

[15] Staël, von., Holstein, C., Eriksson, S., Huldt, B., et al. (1991). Endoscopic screening during 17 years for gastric stump carcinoma. A prospective clinical trial. Scand J Gastroenterol, 26(10), 1020-6.

[16] Fukayama, M. (2010). Epstein-Barr virus and gastric carcinoma, Pathol Int., 60(5), $337-50$.

[17] Ming, S., \& Goldman, H. (1965). Gastric polyps: a histogenetic classification and its relation to carcinoma. Cancer, 18(6), 721-726.

[18] Utsunomiya, J., Maki, T., Iwama, T., et al. (1974). Gastric lesion of familial polyposis coli. Cancer, 34(3), 745-54.

[19] Aarnio, M., Salovaara, R., Aaltonen, L. A., et al. (1997). Features of gastric cancer in hereditary non-polyposis colorectal cancer syndrome. Int J Cancer, 74(5), 551-5.

[20] Tashiro, A., Sano, M., Kinameri, K., et al. (2006). Comparing mass screening techniques for gastric cancer in Japan. World J Gastroenterol, 12(30), 4874-75.

[21] Shimizu, K., Ito, K., Matsunaga, N., et al. (2005). Diagnosis of gastric cancer with MDCT using the water-filling method and multiplanar reconstruction: CT-histologic correlation. AJR Am J Roentgenol, 185(5), 1152-58.

[22] Chen, C. Y., Hsu, J. S., Wu, D. C., et al. (2007). Gastric cancer: preoperative local staging with 3D multi-detector row CT: correlation with surgical and histopathologic results. Radiology, 242(2), 472-82.

[23] Ba-Ssalamah, A., Prokop, M., Uffmann, M., et al. (2003). Dedicated multidetector CT of the stomach: spectrum of diseases. Radio Graphics, 23(3), 625-644.

[24] Kim, Y. H., Lee, K. H., Park, S. H., et al. (2009). Staging of T3 and T4 gastric carcinoma with multidetector CT: added value of multiplanar reformation for prediction of adjacent organ invasion. Radiology, 250(3), 767-775.

[25] Lim, J. S., Yun, M. J., Kim, M. J., et al. (2006). CT and PET in stomach cancer: preoperative staging and monitoring of response to therapy. Radio Graphics 26(1), 143-156.

[26] Kim, S. J., Kim, H. H., Kim, Y. H., et al. (2009). Peritoneal metastasis: detection with 16- or 64-detector row CT in patients undergoing surgery for gastric cancer. Radiolo$g y, 253(2), 407-415$.

[27] Park, S. H., Han, J. K., Kim, T. K., et al. (1999). Unusual gastric tumors: radiologicpathologic correlation. Radio Graphics, 19(6), 1435-1446.

[28] Dickson, A. M., Schuss, A., Goyal, A., et al. (2004). Radiology-Pathology Conference:Calcified untreated gastric cancer. Clin Imaging, 28(6), 418-21. 
[29] Murayama, H., Kamio, A., Imai, T., et al. (1982). Gastric carcinoma with psammomatous calcification: report of a case, with reference to calcinogenesis. Cancer, 49(4), 788-96.

[30] Mei, M., Jingmei, N., Zongming, C., et al. (2012). Diffuse type gastric carcinoma presenting as giant gastric folds: Lessons learned from six miss diagnosed cases. Clin Res Hepatol Gastroenterol, http://dx.doi.org/10.1016/j.clinre,2012.04.009.

[31] Johnson, P. T., Horton, K. M., \& Fishman, E. K. (2010). Hypervascular gastric masses: CT findings and clinical correlates. AJR Am J Roentgenol 2010 Dec. 195(6):W, 415-20.

[32] van Beek, J., zur Hausen, A., Klein Kranenbarg, E., et al. (2004). EBV-positive gastric adenocarcinomas: a distinct clinicopathologic entity with a low frequency of lymph node involvement, J Clin Oncol., 22(4), 664-70.

[33] Lee, J. H., Kim, S. H., Han, S. H., et al. (2009). Clinicopathological and molecular characteristics of Epstein-Barr virus-associated gastric carcinoma: a meta-analysis, $J$ Gastroenterol Hepatol, 24(3), 354-65.

[34] Murphy, G., Pfeiffer, R., Camargo, M. C., et al. (2009). Meta-analysis shows that prevalence of Epstein-Barr virus-positive gastric cancer differs based on sex and anatomic location, Gastroenterology, 137(3), 824-33.

[35] Tokunaga, M., \& Land, C. E. (1998). Epstein-Barr virus involvement in gastric cancer: biomarker for lymph node metastasis, Cancer Epidemiol Biomarkers Prev., 7(5), 449-50.

[36] Fukayama, M., \& Ushiku, T. (2011). Epstein-Barr virus-associated gastric carcinoma, Pathol Res Pract., 207(9), 529-37.

[37] Watanabe, H., Enjoji, M., \& Imai, T. (1976). Gastric carcinoma with lymphoid stroma. Its morphologic characteristics and prognostic correlations, Cancer, 38(1), 232-43.

[38] Maeda, E., Akahane, M., Uozaki, H., et al. (2009). CT appearance of Epstein-Barr virus-associated gastric carcinoma, Abdom Imaging, 34(5), 618-25.

[39] Randjelovic, T., Filipovic, B., Babic, D., et al. (2007). Carcinosarcoma of the stomach: a case report and review of the literature. World J Gastroenterol, 13(41), 5533-6. 